Quim. Nova, Vol. 34, No. 6, 968-972, 2011

\title{
NANOSTRUCTURED SYSTEMS CONTAINING AN ESSENTIAL OIL: PROTECTION AGAINST VOLATILIZATION
}

\author{
Fernanda Cramer Flores, Roseane Fagundes Ribeiro, Aline Ferreira Ourique, Clarice Madalena Bueno Rolim e Cristiane \\ de Bona da Silva* \\ Departamento de Farmácia Industrial, Centro de Ciências da Saúde, Universidade Federal de Santa Maria, AV. Roraima, 1000, \\ 97105-900 Santa Maria - RS, Brasil \\ Adriana Raffin Pohlmann \\ Departamento de Química Orgânica, Instituto de Química, Universidade Federal de Rio Grande do Sul, CP 15003, 91501-970 \\ Porto Alegre - RS, Brasil \\ Ruy Carlos Ruver Beck e Sílvia Stanisçuaski Guterres \\ Faculdade de Farmácia, Universidade Federal do Rio Grande do Sul, Av. Ipiranga, 2752, 90610-000 Porto Alegre - RS, Brasil
}

Recebido em 19/8/10; aceito em 22/12/10; publicado na web em 25/3/11

\begin{abstract}
The goal of this study was to evaluate the feasibility of preparing nanocapsules and nanoemulsions using tea tree oil as oily phase aiming to protect its volatilization. The nanostructures presented nanometric mean size $(160-220 \mathrm{~nm})$ with a polydispersity index below 0.25 and negative zeta potential. The $\mathrm{pH}$ values were $6.43 \pm 0.37$ and $5.98 \pm 0.00$ for nanoemulsions and nanocapsules, respectively. The oil content after preparation was $96 \%$. The inclusion of tea tree oil in nanocapsules showed higher protection against volatilization. The analysis of mean size and polydispersity index of formulations presented no significant alteration during the storage time.
\end{abstract}

Keywords: nanoemulsions; nanocapsules; tea tree essential oil.

\section{INTRODUCTION}

The use of raw materials of plant origin in pharmaceutical products has been growing in the last years. The diversity of medicinal plants, some characterized by the presence of substances with potential pharmacological effect, contributes to several studies that aim to demonstrate the action and effectiveness of some compounds.

Essential oils, obtained from different plant species, have been promising, particularly because of their antimicrobial, insecticides and antiviral properties ${ }^{1,2}$ and they may be incorporated in pharmaceutical preparations for the topical treatment of skin diseases. ${ }^{3}$ However, because of their chemical complexity and susceptibility to degradation, besides volatility and insolubility in water, it is necessary to employ procedures to improve the oil stability contributing to the product's effectiveness.

One essential oil of particular interest over the last years has been the tea tree oil (TTO) obtained from leaves of Melaleuca alternifolia (Myrtaceae) species by a steam distillation process. TTO is popular as a topically applied product due to its known medicinal properties, particularly its antibacterial ${ }^{4-7}$ and antifungal activities. ${ }^{8}{ }^{89}$ It has been used topically to treat conditions such as acne, onychomycosis, oral candidiasis, and tinea pedis. ${ }^{10}$ Additionally, TTO presents antiviral ${ }^{11}$ and anti-inflammatory properties. ${ }^{12}$

TTO contains over 100 components, mainly monoterpenes and sesquiterpenes hydrocarbons and their associated alcohols. Its main component is terpinen-4-ol, followed by $\alpha$-terpinene and $\gamma$-terpinene, which is attributed to the broad spectrum antiseptic activity. ${ }^{9,13,14}$ The physical characteristics of TTO present certain difficulties for the formulation and packaging of products. Its lipophilicity leads

*e-mail: csbona@smail.ufsm.br to miscibility problems in water-based products, while its volatility means that packaging must provide an adequate barrier to volatilization. Moreover, the composition of TTO may change during storage, and light, heat, exposure to air, and moisture can affect its stability. ${ }^{10}$

The TTO in its pure form presents on phototoxic and allergenic potential in relation to topical. ${ }^{15}$ When incorporated in topical formulations, can irritate skin and cause allergic reactions in a dose-dependent individuals predisposed. However, dermal toxicity is not reported in formulations containing $1 \%(\mathrm{v} / \mathrm{v})$ of oil. ${ }^{16}$ The occurrence of allergic reactions and irritation is related to oxidation of the components present in the oil depending on storage conditions and packaging material used. The essential oil is absorbed in plastic, affecting thus its stability. ${ }^{10}$

Different carrier systems have been extensively studied with the aim of controlling the drug release and improving the efficacy and selectivity of drugs. ${ }^{17,18}$ Nanocapsules, nanospheres, nanoemulsions, and other nanometric systems have a great surface area, which makes them suitable for important pharmaceutical and cosmetic applications, such as topical aqueous formulations of lipophilic encapsulated drugs for a homogeneous release..$^{19}$ An important advantage of these systems is their small size (below $1 \mu \mathrm{m}$ ), which facilitates their formulation in dermatological products and enables comfortable application to the skin. ${ }^{19,20}$ Besides, the possibility of increasing the effectiveness and stability of formulations or active substances, as well as their gradual release in adequate doses are good advantages too. ${ }^{21,22}$

This way, this work aims to propose a strategy to prepare nanoemulsions and polymeric nanocapsules formulations using TTO as oily phase due its interesting properties for topical application. Besides the physicochemical characteristics, we evaluated the potential of these systems to improve the TTO stability in terms of volatilization. 


\section{EXPERIMENTAL}

\section{Materials}

Tea tree essential oil (Melaleuca alternifolia) was obtained from Laszlo Aromaterapia (Belo Horizonte, Brazil). Poly( $\varepsilon$-caprolactone) $(\mathrm{Mw}=80,000)$ and sorbitan monooleate were acquired from Sigma (São Paulo, Brazil). Polysorbate 80 was supplied by Delaware (Porto Alegre, Brazil). All other chemicals and solvents were of pharmaceutical grade and were used as received.

\section{GC analysis}

The levels of the major components of TTO were determined by gas chromatography (Departamento de Química, Universidade Federal de Minas Gerais). GC-FID was carried out on a chromatograph (Varian CP3380), using a fused silica capillary column (DB5 $30 \mathrm{~m} \times 0.25 \mathrm{~mm}$, $\mathrm{J} \& \mathrm{~W}$ Scientific) with hydrogen carrier gas $\left(1.8 \mathrm{~mL} \mathrm{~min}^{-1}\right)$. The temperature programming ranged from $50(3 \mathrm{~min})$ to $160{ }^{\circ} \mathrm{C}$ at $3{ }^{\circ} \mathrm{C} \mathrm{min}{ }^{-1}$ increments. The FID injector and detector temperatures were $200^{\circ} \mathrm{C}$. The oil was diluted in chloroform $(0.5 \%)$ and $1 \mu \mathrm{L}$ was injected. Identification and quantification of components was made by calculating the retention indices and normalization of the area, respectively.

\section{Swelling experiments}

Films of poly( $\varepsilon$-caprolactone) were obtained by squeezing of a hydraulic press for $5 \mathrm{~min}$ at 5 Tons (Hydraulic Press P30000, Bovenau, Brazil). Each film was exactly weighed and than immersed in sufficient volume $(2 \mathrm{~mL})$ of TTO in different flasks $(\mathrm{n}=3)$. The flasks were closed and stored at room temperature. At predetermined time intervals $(3,5,10,15,30$ and 60 days $)$, the films were pinched and TTO was removed using absorbing paper, thereafter the films were weighed again. ${ }^{23}$

\section{Preparation of the formulations}

Our strategy to prepare nanoemulsions and nanocapsules suspensions containing an essential oil using known methods (spontaneous emulsification and interfacial deposition of preformed polymer) was based on the optimization of the volume of both phases during the preparation (aqueous and organic phases). In order to avoid the steam distillation of TTO during the evaporation step, the volume of water was fixed to a minimum.

Nanoemulsions (NE) and nanocapsules suspensions (NC) were prepared $(n=3)$ by spontaneous emulsification ${ }^{19}$ and interfacial deposition of preformed polymer method, ${ }^{24}$ respectively. Briefly, an organic phase composed of TTO $(0.5 \mathrm{~g})$, sorbitan monooleate $(0.383 \mathrm{~g})$, poly ( $\varepsilon$-caprolactone) $(0.25 \mathrm{~g})$, and acetone $(25.0 \mathrm{~mL})$ was added to an aqueous solution $(50.0 \mathrm{~mL})$ containing polysorbate 80 $(0.383 \mathrm{~g})$ and kept under moderate magnetic stirring for $10 \mathrm{~min}$. Then, the organic solvent was eliminated in rotary evaporator (Fisatom, São Paulo, Brazil) at $60 \mathrm{rpm}$ and $30-35^{\circ} \mathrm{C}$ temperature. The final volume of the formulations was fixed in $50 \mathrm{~mL}$ to obtain an oil concentration of $1 \%$ of oil $(10 \mathrm{mg} / \mathrm{mL})$. NE were prepared omitting the polymer. As a control, an emulsion (coarse dispersion) was prepared omitting the organic solvent and the lipophilic surfactant.

\section{Characterization of formulations}

Particle size, polydispersity indices and zeta potential analysis

The particle sizes and polydispersity indices $(n=3)$ were measured by photon correlation spectroscopy after adequate dilution of an aliquot of the samples in purified water (Zetasizer Nanoseries, Malvern Instruments, Worcestershire, UK). The zeta potential values were measured using the same instrument at $25^{\circ} \mathrm{C}$, after dilution of the samples in $10 \mathrm{mM} \mathrm{NaCl}$.

\section{pH determination}

The $\mathrm{pH}$ values of the $\mathrm{NE}$ and $\mathrm{NC}$ were determined directly in the samples using a calibrated potentiometer (Mettler Toledo, São Paulo, Brazil), at room temperature.

\section{Determination of oil content}

The total oil content (\%) in the NE, NC and emulsion formulations was determined in triplicate by distilling $25 \mathrm{~mL}$ of samples for $3 \mathrm{~h}$ in a Clevenger's apparatus. For NC, after initial extraction, $20 \mathrm{~mL}$ of acetonitrile was added into extraction flask, and this solution was evaporated for $1 \mathrm{~h}$ more. The volume of TTO collected in the trap was weighted to calculate the oil recovered from the sample.

\section{Morphological analyses}

Morphological analyses were development by transmission electron microscopy (TEM; Jeol, JEM 1200 Exll, Centro de Microscopia - UFRGS), operating at $80 \mathrm{kV}$. The samples (NE and NC) were diluted and deposited in Form-Carbon support films on specimen grid (Electron Microscopy Sciences), negatively stained with uranyl acetate solution $(2 \% \mathrm{w} / \mathrm{v})$ and observed at 150,000 of magnification.

\section{Study of protection of the essential oil volatilization}

The influence of the nanoencapsulation against essential oil evaporation was evaluated at $37 \pm 1{ }^{\circ} \mathrm{C}$. Samples (NE, NC and emulsion) were heated on a water-bath for 15,30 and $60 \mathrm{~min}$. After, the content of the essential oil was determined by hydro-distillation, distilling $25 \mathrm{~mL}$ of samples for $3 \mathrm{~h}$ in a Clevenger's apparatus $(\mathrm{n}=$ 3 ). The volume of TTO was collected and weighted to calculate the oil recovered from the sample.

\section{Stability studies}

Formulations were monitored following preparation until 2 months of storage by mean size, polydispersity, zeta potential and $\mathrm{pH}$. The oil content was determined after 1 month of storage. Formulations were packaged in amber glass containers and stored at room temperature $\left(25 \pm 2{ }^{\circ} \mathrm{C}\right)$ and protected from light.

\section{Statistical analysis}

Formulations were prepared and analyzed in triplicate. Results are expressed as mean \pm SD (standard deviation). One-way analysis of variance (ANOVA) was employed in the comparison of the experimental data, using the SigmaStat Statistical Program (Version 3.0, Jandel Scientific, USA).

\section{RESULTS AND DISCUSSION}

\section{GC analysis}

TTO composition is regulated by an international standard (ISO 4730) for Oil of Melaleuca - terpinen-4-ol type, which sets maxima and/or minima for 14 components of the oil. ${ }^{10}$ The oil used in the study was analyzed and shown to contain acceptable levels of mean components: terpinen-4-ol (39.4\%), $\gamma$-terpinene (22.9\%), $\alpha$-terpinene (10.2\%), 1,8-cineole $(5.1 \%)$, terpinolene $(3.6 \%)$, $\rho$-cymene $(3.4 \%)$, $\alpha$-pinene $(3.2 \%)$ and $\alpha$-terpineol $(1.9 \%)$. 


\section{Swelling experiment}

The objective of this experiment was to determinate the possible solubilization of poly( $\varepsilon$-caprolactone) by TTO. In this case, the polymeric wall of nanocapsules could be dissolved by the oily core resulting in the formation of nanometric oily droplets (nanoemulsion) during the storage time. The results showed that the polymer weights have a small mass gain during the first three days of the experiment (9.8\% in relation to the initial weight) After, the weight of polymer remained constant until the 60 days of the experiment (Figure 1), indicating that no solubilization of poly( $\varepsilon$-caprolactone) occurred by the oil. No statistical difference was observed between the polymer weights during all the time of the experiment $(p>0.05)$. This result shows that TTO and poly( $\varepsilon$-caprolactone) have low interactions at the macroscopic scale, indicating that after preparing of nanocapsules with TTO the polymeric wall will not be dissolved, maintaining the required characteristics.

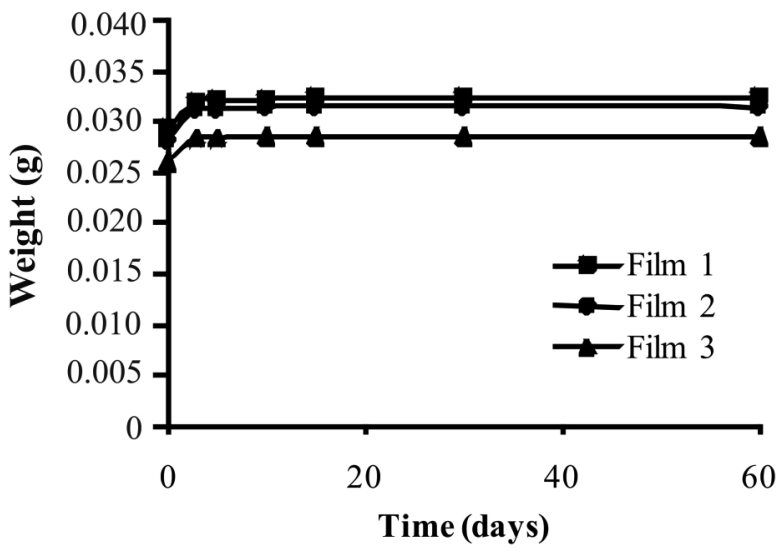

Figure 1. Swelling experiment: films weight at time intervals

\section{Physicochemical properties of formulation}

The NE and NC containing TTO appeared macroscopically homogeneous and opalescent bluish liquids. The physicochemical characteristics of the formulations are presented in Table 1. TTOloaded nanocapsules and nanoemulsions presented nanometric mean diameters (160-220 $\mathrm{nm}$ ) as well as polydispersity indices below 0.25 indicating an adequate homogeneity of these systems. The values obtained are in agreement with those usually reported for these systems $(100-500 \mathrm{~nm}){ }^{25-27}$ The formulations showed acid $\mathrm{pH}(5.9-6.5)$ and negative zeta potentials (about $-13 \mathrm{mV}$ ). The negative zeta potential values presented by the samples are related to the presence of polysorbate 80 , presenting a negative surface density of charge due to the presence of oxygen atoms in the molecules. ${ }^{28}$ Moreover, the type of oily phase used in the preparation of the colloidal systems may be influenced the values (in module) obtained. ${ }^{17}$ Ourique and co-workers ${ }^{26}$ reported zeta potential values of -4.45 and -5.14 for nanocapsules and nanoemulsions prepared with the capric/caprylic triglyceride mixture, and -8.38 and -6.13 for nanocapsules and nanoemulsions containing sunflower seed oil. TEM analysis showed homogeneous and spherical particles with similar diameters as determined by photon correlation spectroscopy (Figure 2).

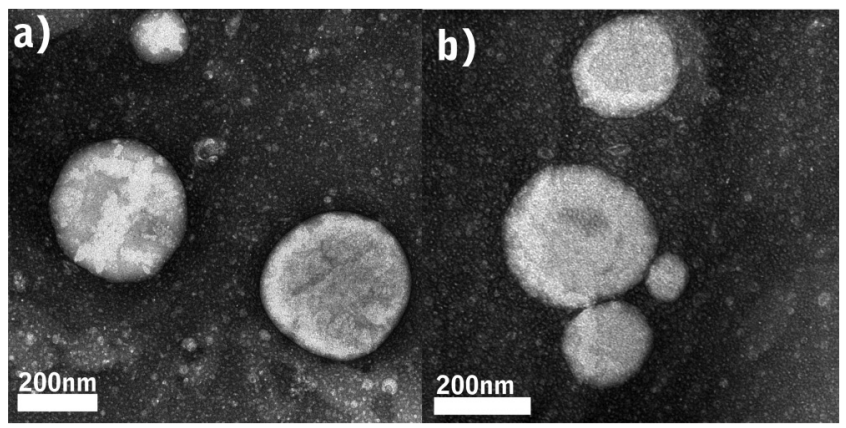

Figure 2. TEM images of $N C(a)(150,000 x ;$ bar $=200 \mathrm{~nm})$ and $N E(b)$ $(150,000 x ;$ bar $=200 \mathrm{~nm})$

The yield of oil obtained was $96.0 \pm 0.3 \%$ for NE and $95.7 \pm$ $0.6 \%$ for NC (Table 1) of the theoretical concentration, being that only $4 \%$ of the oil was lost during the preparation, probably during the evaporation of organic solvent. This explanation can be reinforced by the results obtained for the coarse emulsions prepared without the evaporation step, presenting an oil content of $99.2 \pm 0.2 \%$.

Another approach to encapsulate an essential oil (turmeric oil) was studied by Lertsutthiwong and co-workers. ${ }^{29,30}$ They used biopolymers (alginate and chitosan-alginate) and nanocapsules were prepared in three-step procedure (emulsification, gelification, and solvent removal). The results showed that sonication was required to obtain nanocapsules with uniform size, and about $42 \%$ of the turmeric oil was lost during the preparation of alginate nanocapsules. ${ }^{29}$ The addition of chitosan to the formulations led to a lower oil less during the preparation (percentage recovery from 61.9-68.5\%).$^{30}$ Regarding our work, the interfacial deposition of preformed polymer method was suitable and presented advantages to obtain nanocapsules and nanoemulsions containing an essential oil (TTO) due to the obtaining of uniform particle size, the oil recovery above $90 \%$ and the lesser time for the preparation (two-step procedure).

TTO as well as other essential oils have a pronounced odor that sometimes should be masked in formulations. This way, we analyzed the odor of our formulations, comparing the intensity of their odor with the pure essential oil. The odor intensity was $\mathrm{NC}<\mathrm{NE}<$ emulsion $<$ pure oil. It is important to point out that the incorporation of TTO in nanocapsules allows reducing considerably the odor of the oil. This result is in agreement with the ability of polymeric nanocapsules to mask physicochemical properties of some substances. ${ }^{22}$

\section{Protection of the essential oil volatilization}

In this test, differences between the formulations were observed. After $60 \mathrm{~min}$, the oil content was 30,33 and 54\% in relation to the initial values for emulsion, $\mathrm{NE}$ and $\mathrm{NC}$, respectively. The oil content in $\mathrm{NC}$ was about $67 \%$ after 30 min of heating; values lower than 42 and $40 \%$ were obtained for NE and emulsion, respectively (Figure 3 ).

Table 1. Physicochemical characteristics of colloidal systems (NE and NC) prepared with TTO (mean \pm S.D., represents the variation between the 3 different batches)

\begin{tabular}{lccccc}
\hline Formulation & Particle size $(\mathrm{nm})$ & PDI* $^{*}$ & Zeta potential $(\mathrm{mV})$ & Oil content $(\%)$ & $\mathrm{pH}$ \\
\hline $\mathrm{NE}$ & $167 \pm 04$ & $0.17 \pm 0.01$ & $-13.3 \pm 2.6$ & $96.0 \pm 0.3$ & $6.43 \pm 0.37$ \\
$\mathrm{NC}$ & $212 \pm 08$ & $0.17 \pm 0.01$ & $-13.5 \pm 1.1$ & $95.7 \pm 0.6$ & $5.98 \pm 0.00$ \\
\hline
\end{tabular}

*PDI: polydispersity index. 
Table 2. Stability studies: physicochemical characteristics of NE and NC containing TTO (1\%) after 1 and 2 months of storage at room temperature and protected from light (mean $\pm \mathrm{SD}, \mathrm{n}=3$ )

\begin{tabular}{|c|c|c|c|c|c|}
\hline Time (months) & Particle size (nm) & PDI* & Zeta potential (mV) & $\mathrm{pH}$ & Oil content (\%) \\
\hline \multicolumn{6}{|l|}{$\mathrm{NE}$} \\
\hline 1 & $166 \pm 07$ & $0.17 \pm 0.03$ & $-9.6 \pm 1.8$ & $5.10 \pm 0.15$ & $91.0 \pm 1.0$ \\
\hline 2 & $166 \pm 06$ & $0.16 \pm 0.02$ & $-14.6 \pm 3.8$ & $5.01 \pm 0.10$ & ND \\
\hline \multicolumn{6}{|l|}{$\mathrm{NC}$} \\
\hline 1 & $210 \pm 09$ & $0.19 \pm 0.03$ & $-12.6 \pm 2.1$ & $5.14 \pm 0.17$ & $90.0 \pm 0.3$ \\
\hline 2 & $211 \pm 05$ & $0.17 \pm 0.02$ & $-18.8 \pm 1.7$ & $4.91 \pm 0.18$ & ND \\
\hline
\end{tabular}

"PDI: polydispersity index; ND: not determined.

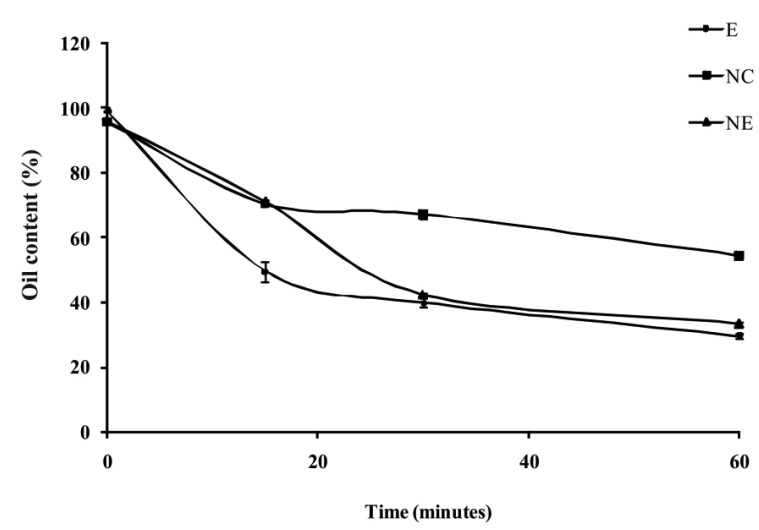

Figure 3. Oil content in the $N E, N C$ and emulsion after heat at $37^{\circ} \mathrm{C}$, during 15, 30 and $60 \mathrm{~min}$

These results showed the potential of NC in protecting oil against evaporation, improving its stability. This protection can be attributed to the presence of the polymeric wall in the nanocapsules suspensions, avoiding the volatilization of the oil and its contact with the aqueous phase. Thus, it can be suggest that oxidation reactions and degradation of compounds can be minimized by improving the stability and safety of TTO preparations. Moreover, NE also showed a protection against oil volatilization compared to the coarse emulsions, although this protection was lower than that observed for NC.

\section{Stability studies}

Regarding preliminary stability studies, no statistical difference was observed for the values of mean size and polydispersity index of formulations during the storage. However, an increased in zeta potential values (in module) was observed (Table 2).

Schaffazick and co-workers ${ }^{17}$ report that the zeta potential is influenced by changes in the interface with the dispersing medium, due to the dissociation of functional groups on the surface of the particle, depending on the relaxation of the polymer chain over time. On the other hand, the $\mathrm{pH}$ values presented a decrease both $\mathrm{NC}$ and $\mathrm{NE}$ after 2 months of storage ( $\mathrm{p} \leq 0.05$ ). This decrease, for $\mathrm{NE} \mathrm{pH}$ values, may be related to the higher contact of the essential oil with the aqueous phase leading, for hypothesis, to the degradation of some components as observed by their lesser protection against oil volatilization compared to $\mathrm{NC}$. The decrease in the $\mathrm{pH}$ values to $\mathrm{NC}$ could be explained by the polymer chains relaxations, which exposes a higher number of terminal carboxylic groups. ${ }^{17}$ These results are in concordance with others works reported. ${ }^{31}$ Is important to relate that this reduction was minor that one unit after 1 mouth of storage for NC. Additionally, the oil content after 30 days storage was evaluated, and the yield obtained was above $90 \%$ for both NC and NE (Table 2). Additionally, during the stability study, the odor of the formulations remained the same for both formulations ( $\mathrm{NC}$ and $\mathrm{NE}$ ).

\section{CONCLUSION}

In this work, we proposed a strategy to obtain nanocapsules suspensions and nanoemulsions containing TTO due to its important pharmacological properties already reported in literature. The formulations presented good physicochemical characteristics related to their nanostructured character and adequate physicochemical stability. Moreover, the incorporation of TTO in nanocapsules protected it from evaporation, improving its stability and decreasing its pronounced odor. These results allow us to suggest the use of this strategy as a platform to prepare nanostructured systems containing essential oils. Studies are in progress to evaluate the antimicrobial activity of these systems as well as their incorporation in semisolid formulations as alternatives to topical delivery of TTO.

\section{ACKNOWLEDGEMENTS}

The authors thank the financial support of CNPq-Brasília/Brazil and Rede Nanocosméticos/CNPq.

\section{REFERENCES}

1. Burt, S.; Int. J. Food Microbiol. 2004, 94, 223.

2. Bakkali, F.; Averbeck, S.; Averbeck, D.; Idaomar, M.; Food Chem. Toxicol. 2008, 46, 446.

3. Harris, R.; Int. J. Aromather. 2002, 12, 83.

4. Raman, A.; Weir, U.; Bloomfield, S. F.; Lett. Appl. Microbiol. 1995, 21 , 242.

5. Cox, S. D.; Mann, C. M.; Markham, J. L.; Bell, H. C.; Gustafson, J. E.; Warmington, J. R.; Wyllie, S. G.; J. Appl Microbiol. 2000, 88, 170.

6. Carson, C. F.; Mee, B. J.; Riley, T. V.; Antimicrob. Agents Chemother. 2002, 46, 1914.

7. Halcón, L.; Milkus, K.; Am. J. Infect. Control 2004, 32, 402.

8. Hammer, K. A.; Carson, C. F.; Riley, T. V.; J. Antimicrob. Chemother. 2002, 50, 195

9. Hammer, K. A.; Carson, C. F.; Riley, T. V.; J. Appl. Microbiol. 2003, 95 , 853.

10. Carson, C. F.; Hammer, K. A.; Riley, T. V.; Clin. Microbiol. Rev. 2006, 19,50 .

11. Schnitzeler, P.; Schön, K.; Reichling, J.; Pharmazie 2001, 56, 343.

12. Hart, P. H.; Brand, C.; Carson, C. F.; Riley, T. V.; Prager, R. H.; FinlayJones, J. J.; Inflamm. Res. 2000, 49, 619.

13. Carson, C. F.; Riley, T. V.; Lett. Appl. Microbiol. 1995, 19, 24.

14. Oliva, B.; Piccirilli, E.; Ceddia, T.; Pontieri, E.; Aureli, P.; Ferrini, A. M.; Lett. Appl. Microbiol. 2003, 37, 185.

15. Carson, C. F.; Riley, T. V.; Cookson, B. D.; J. Hospital Infection 1998, 40,175 .

16. Hammer, K. A.; Carson, C. F.; Riley, T. V.; Nielsen, J. B.; Food Chem. Toxicol. 2006, 44, 616.

17. Schaffazick, S. R.; Guterres, S. S.; Freitas, L. L.; Pohlmann, A. R.; Quim. Nova 2003, 26, 726. 
18. Ravi Kumar, M. N. V.; Kumar, N.; Drug Develop. Ind. Pharm. 2001, $27,1$.

19. Bouchemal, K.; Briançon, S.; Perrier, E.; Fessi, H.; Int. J. Pharm. 2004, 280, 241.

20. Perugini, P.; Simeoni, S.; Scalia, S.; Genta, I.; Modena, T.; Conti, B.; Pavanetto, F.; Int. J. Pharm. 2002, 246, 37.

21. Barratt, G. M.; Pharma. Sci. Technol. Today 2000, 3, 163.

22. Guterres, S. S.; Alves, M. P.; Pohlmann, A. R.; Drug Target Insights 2007, 2, 147.

23. Weiss-Angeli, V.; Polleto, F. S.; Zancan, L. R.; Baldasso, F.; Pohlmnan, A. R.; Guterres, S. S.; J. Biom. Nanotechnol. 2008, 4, 80.

24. Fessi, H.; Puisieux, F.; Devissaguet, J. P.; EP 0274961 A1, 1988.

25. Friedrich, R. B.; Camponogara, M. F.; Beck, R. C. R.; Pohlmann, A. R.; Guterres, S. S.; Quim. Nova 2008, 31, 1131.
26. Ourique, A. F.; Pohlmann, A. R.; Guterres, S. S.; Beck, R. C. R.; Int. J. Pharm. 2008, 352, 1.

27. Fontana, M. C.; Coradini, K.; Guterres, S. S.; Pohlmann, A. R.; Beck, R. C. R.; J. Biomed. Nanotech. 2009, 5, 1.

28. Marchiori, M. L.; Lubini, G.; Dalla Nora, G.; Friedrich, R. B.; Fontana, M. C.; Ourique, A. F.; Bastos, M. O.; Rigo, L. A.; Silva, C. B.; Tedesco, S. B.; Beck, R. C. R.; Drug Develop. Ind. Pharm. 2010, 36, 962.

29. Lertsutthiwong, P.; Noomun, K.; Jongaroonngamsang, N.; Rojsitthisak, P.; Nimmannit, U.; Carbohydr. Polym. 2008, 74, 209.

30. Lertsutthiwong, P.; Rojsitthisak, P.; Nimmannit, U.; Mater. Sci. Eng., C 2009, 29, 856.

31. Jager, A.; Stefani, V.; Guterres, S. S.; Pohlmann, A. R.; Int. J. Pharm. 2007, 338, 297 